\title{
Surgery and MRI increase the risk of hypothermia in infants.
}

Joel M Don Paul ${ }^{1,2}$, Elizabeth J Perkins ${ }^{1}$, Prue M Pereira-Fantini ${ }^{1}$, Asha Suka ${ }^{1,2}$, Olivia Farrell $^{1,2}$, Julia K Gunn ${ }^{1,2,3}$, Anushi E Rajapaksa ${ }^{1,2}$, David G Tingay ${ }^{1,2,3}$

${ }^{1}$ Neonatal Research, Murdoch Childrens Research Institute, Melbourne, Australia

${ }^{2}$ Department of Paediatrics, University of Melbourne, Melbourne, Australia

${ }^{3}$ Neonatology, Royal Children's Hospital, Melbourne, Australia

\section{Corresponding Author:}

A/Prof David G Tingay MB BS DCH FRACP PhD.

Neonatal Research

Murdoch Children's Research Institute

Royal Children's Hospital Flemington Rd.

Parkville 3052 Victoria

Australia

$+61393454023$

+61393455067 (fax)

Email: david.tingay@rch.org.au

Short title: Hypothermia during surgery and MRI

Word Count: 2660 words

Abstract Word Count: 250 words

This is the author manuscript accepted for publication and has undergone full peer review but has not been through the copyediting, typesetting, pagination and proofreading process, which may lead to differences between this version and the Version of Record. Please cite this article as doi: $10.1111 /$ jpc.13824

This article is protected by copyright. All rights reserved. 


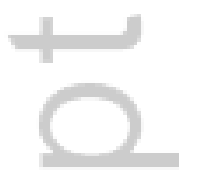


Author Contributions: DGT developed the concept and designed the experiment. JDP, AS and EJP enrolled and studied all infants. All authors were involved in data analysis. JDP, AS, PP-F, JKG and DGT interpreted the data. JDP, PP-F and DGT wrote the first draft of the manuscript and all authors contributed to redrafting the manuscript.

Financial Support: This study is supported by the Victorian Government Operational Infrastructure Support Program (Melbourne, Australia). DGT is supported by a National Health and Medical Research Council Clinical Career Development Fellowship (Grant ID 1053889).

Competing Interests: The authors have no competing interests to declare. No author received an honorarium, grant, or other form of payment to produce the manuscript. The study was not commissioned and no commercial agencies were involved in any aspect of this study. 


\section{Surgery and MRI increase the risk of hypothermia in infants.}

\section{ABSTRACT}

Background: Maintaining normothermia is a tenet of neonatal care. However, neonatal thermal care guidelines applicable to intra-hospital transport beyond the neonatal intensive care unit (NICU) and during surgery or magnetic resonance imaging (MRI) are lacking.

Aims: To determine the proportion of infants normothermic $\left(36.5^{\circ} \mathrm{C}-37.5^{\circ} \mathrm{C}\right)$ on return to NICU after management during surgery and MRI, and during standard clinical care in both environments.

Methods: Sixty two newborn infants requiring either surgery in the operating theatre (OT) $(n=41)$ or an MRI scan $(\mathrm{n}=21)$ at the Royal Children's Hospital (Melbourne) NICU were prospectively studied. Core temperature, along with cardiorespiratory parameters, was continuously measured from 15 minutes prior to leaving the NICU until 60 minutes after returning. Passive and active warming (intra-operatively) was at clinician discretion.

Results: $90 \%$ of infants were normothermic before leaving NICU; 86\% (MRI) and 93\% (OT). Only $52 \%$ of infants were normothermic on return to NICU; RR $(95 \% \mathrm{CI}) 1.75(1.39,2.31)$, NNH 2.6. Between departure from the NICU and commencement of surgery, core temperature decreased by mean $(95 \% \mathrm{CI}) 0.81(0.30,1.33)^{\circ} \mathrm{C}(\mathrm{p}=0.0001$, ANOVA), with only $24 \%$ of infants normothermic when surgery began $(\mathrm{p}<0.0001)$; RR $(95 \% \mathrm{CI}) 3.80(2.33,6.74), \mathrm{NNH} 1.5$. After an MRI, infants were a mean $(95 \% \mathrm{CI}) 0.41(0.16,0.67)^{\circ} \mathrm{C}$ colder than immediately before entering the scanner $(p=0.001$, ANOVA), with only $43 \%$ being normothermic; $p=0.003$, RR $(95 \% \mathrm{CI}) 2.11(1.35,3.74)$, NNH 2.1.

Conclusions: Unintentional hypothermia is a common occurrence during surgery in the OT and MRI in neonates, indicating that evidence-based warming strategies to prevent hypothermia should be developed.

Keywords: Infant; MRI; normothermia; hypothermia; surgery; temperature regulation. 


\section{Key Points}

1. Newborns are highly susceptible to inadvertent heat loss when ambient temperatures are lower than core temperature.

2. Hypothermia is a frequent occurrence during pre-surgery perioperative care and during a MRI scan.

3. Hypothermia was present in $50 \%$ of neonates on return to NICU post procedure and persisted despite access to normal neonatal nursing practices.

\section{Multiple Choice Questions}

1. Which of the following reduces the risk of inadvertent hypothermia?
A. Ambient room temperature above $25^{\circ} \mathrm{C}$
B. Polyurethane wraps
C. Bair Hugger
D. All of the above

Answer: D. All of the above have been shown to reduce hypothermia in the Delivery Room and Operating Theatre. Neonates are at risk from heat loss via multiple mechanisms, including radiation, convection, conduction and evaporation. Warming mechanisms can be considered passive (eg polyurethane wraps and room temperature) or active (Bair Hugger). Only polyurethane wraps can be used in MRI.

2. Which of the following mechanisms of heat loss is less likely during an MRI than in the Operating Theatre or Delivery Room?
A. Conduction
B. Radiation
C. Evaporation
D. Convection

Answer: C. Evaporation refers to the loss of heat moving from a warm to cooler region via the latent heat properties of water. Unlike the Delivery Room and Operating Theatre (skin cleaning solutions, exposed organs) infants are rarely wet during an MRI.

3. Infants are at increased risk of hyperthermia during an MRI.
A. True
B. False

Answer: B. Previous concerns have been raised that patients may be at risk of hyperthermia during MRI, but we found that the risk of hypothermia after an MRI is twice that before an infant enters 
the scanner room. This is explained by the need to keep the MRI room cooler and the inability to use standard heating systems within the magnetic MRI environment.

This article is protected by copyright. All rights reserved. 


\section{INTRODUCTION}

Neonates are at particular risk of unintentional hypothermia, which is associated with significant harmful consequences leading to increased morbidity and mortality. ${ }^{1-4}$ Accordingly, thermoregulatory management has long been a central tenet of neonatal intensive care, ${ }^{5,6}$ with a myriad of solutions available. In hospital settings outside of the NICU and delivery room (DR), thermoregulatory care has not been emphasised. ${ }^{7}$ In the DR, preventing unintentional hypothermia using both active and passive mechanisms of minimising heat loss has been shown to improve outcomes, with resultant development of evidence-based guidelines and specific practices (such as polyurethane bags for preterm infants). ${ }^{8-11}$

Modern NICU care requires infants to be transported to and managed in the operating theatre (OT) or Magnetic Resonance Imaging (MRI). Both environments pose similar thermal risks as the DR; including ambient temperatures of $<25^{\circ} \mathrm{C}{ }^{12}$ Thermal care options suitable for the NICU are not appropriate in the OT and MRI. Isolettes are impractical for use in the OT, and metallic restrictions in MRI limit active warming systems to expensive systems like MRI-compatible incubators. ${ }^{13}$ Traditionally, neonatal thermal care has been limited to the use of generic warm-air mattresses, such as the Bair Hugger (Arizant Healthcare, Eden Prairie, MN) in the OT and blankets during MRI. ${ }^{14,15}$ Unlike the DR, no evidence-based or standardised thermal guidelines exist for neonates in either environment.

The development of thermal solutions to address neonatal care in the OT and MRI first requires quantifying the degree of temperature instability to which infants are exposed in either environment. To date, this has not been reported. We hypothesised that management in, and transport to the OT or MRI, would expose neonates to similar thermal challenges as the NICU and increase the risk of hypothermia without intentional thermal care. This prospective observational study aimed to determine the thermal patterns during transport and management of neonates in the OT or during MRI. The primary outcome was the incidence of normothermia (defined as a core temperature $36.5^{\circ} \mathrm{C}-37.5^{\circ} \mathrm{C}$ ) on return to the NICU after either surgery in the OT or MRI. Normothermia rates during each procedure and absolute temperature $\left({ }^{\circ} \mathrm{C}\right)$ were secondary measures. 


\section{METHODS}

The study was performed at the Royal Children's Hospital (RCH), Melbourne, Australia, a large regional tertiary referral paediatric hospital for neonatal surgery and neuro-critical-care. The RCH Human and Research Ethics Committee approved the study (HREC35085A and HREC36057A) in accordance with the National Health and Medical Research Council (Australia) guidelines. Written informed consent was obtained from parents prior to enrolment.

Neonates who were inpatients on the NICU (ambient room temperature $25-28^{\circ} \mathrm{C}$ ), less than 50 weeks corrected gestational age at the time of study (April 2015 to September 2016) and had no contraindications to either rectal or oesophageal core temperature monitoring were eligible if they required either surgery in the OT or an MRI $\left(22-25^{\circ} \mathrm{C}\right.$ ambient temperatures), located $130 \mathrm{~m}$ and $120 \mathrm{~m}$ respectively from NICU, and on different floors. Infants were not studied if they had an imperforate anus, necrotising enterocolitis (NEC), Hirschsprung's disease, urological infection or were receiving therapeutic hypothermia. Patients undergoing surgery on the NICU (preterm infants and those on advanced ventilation modalities) were not enrolled. An MRI-compatible transport incubator was not available in our institution at the time of this study.

At least 15 minutes prior to departing the NICU, the thermistor in clinical use at the RCH (Mon-ATherm temperature probe, Covidien, Mansfield, MA) was placed either in the rectum (default) or oesophagus by the bedside clinical team and temperature displayed on a portable monitor (MMS X2 Intellivue, Philips Healthcare, Eindhoven, The Netherlands). Core temperature was then monitored until 60 minutes after return to the NICU, unless the thermistor needed to be removed for clinical reasons. Specifically, the metallic thermistor was removed immediately prior to entering the MRI scanner and then reinserted on leaving the scanner. Heart rate, peripheral oxygen saturation $\left(\mathrm{SpO}_{2}\right)$, mean arterial blood pressure and respiratory rate were also measured at the clinical team's discretion. A NICU nurse transported all infants, with a doctor (OT group) and other staff as required. Infants were transported in an incubator, radiant warmer or open cot without active heat (no internal batteries) at clinical discretion. Humidified and heated gas was used during surgery but was not available during transport. Clinician staff decided on any other thermal interventions used during the study, which in our institution was limited to a Bair Hugger mattress for all infants during surgery and soft blankets during MRI for some infants. 
A researcher accompanied each enrolled infant during the study period and recorded data on a dedicated manual data sheet at standardised time points from 15 minutes before leaving the NICU until 60 minutes after returning. Recorded data included infant core temperature and physiological measures detailed earlier. Surgery was defined as the first skin incision and completion at final skin closure. MRI was defined as the period in which infants were in the restricted metallic zone of the scanner. The MMS X2 monitor displays data continuously and is stored at 12 second intervals for later retrieval. Core temperature and physiological data were then determined at specific management epochs defined as pre-departure and return from NICU, arrival at and departure from OT/MRI, immediate pre- and post-operative care (OT group), pre- and post-entering MRI scanner and start and end of MRI (MRI group). Demographic information for each participant including age, gestation, weight, surgical procedure or body system imaged, relevant medication, any respiratory support ventilation type (if applicable) and any thermal interventions were also recorded.

A sample of 60 infants would detect a clinically meaningful reduction in normothermia of $35 \%$ assuming 90\% normothermia before departing NICU (80\% power, 0.05 alpha error). Based on pilot data from our Institute, this would require recruitment of 40 infants needing surgery and 20 infants undergoing MRI. As incidence of normothermia after OT or MRI was unknown, we received approval to recruit 70 infants. Descriptive data were determined for each phase. Differences in normothermia rates between phases were analysed using Fisher's exact test and a relative risk (RR). Continuous data were tested for normality with temperature and physiological data differences compared using Welch's corrected t-tests, Mann-Whitney tests or one-way repeated measures ANOVA as appropriate. Statistical analysis was performed using GraphPad Prism version 7.01 (GraphPad Software, La Jolla, CA). A p-value of $<0.05$ was considered significant.

\section{RESULTS}

Sixty seven infants were enrolled and studied; 46 were assigned to the OT group and 21 to the MRI group. Five infants in the OT group were excluded due to incomplete temperature recordings at predeparture or arrival to NICU (2 faulty thermistor cable, 3 incorrectly placed probe). The characteristics of the final cohort of 62 infants (41 OT and 21 MRI) are summarised in Table 1. Corrected gestational age (completed weeks) was comparable for infants studied in the OT and MRI groups while the MRI group was a mean $(95 \% \mathrm{CI}) 576(221,930)$ grams heavier at birth $(\mathrm{p}=0.002$, Welch's corrected t-test). Gastrointestinal surgery $(n=24)$ was the most common surgery for those 
infants transported to OT with the OT group spending a median (range) $185(34,355)$ minutes away from the NICU. All infants in the MRI group had imaging of the brain with a median (range) 69 $(45,106)$ minutes away from the NICU. Rectal temperature monitoring was solely used in 53 infants, 3 infants received oesophageal monitoring and 6 a combination in the OT group. Method of temperature monitoring did not influence results. Single journey OT and MRI transport times were median (range) $15(2,56)$ and $5(2,9)$ minutes respectively.

On return to the NICU, $52 \%(n=32)$ of infants were normothermic, compared to $90 \%(n=56)$ prior to leaving (Table 2); $\mathrm{p}<0.0001$, RR $(95 \% \mathrm{CI}) 1.75$ (1.39,2.31), Number Needed to Harm (NNH) 2.6 (Fisher's exact test). By $15 \mathrm{~min}$ after returning to the NICU, only $45 \%$ of infants were normothermic. In the 32 infants with core temperature data $60 \mathrm{~min}$ after return $(85 \%$ OT and $42 \%$ MRI), 69\% were normothermic. Overall, infants became significantly hypothermic immediately before their procedure, with 47\% being normothermic; p<0.0001, RR (95\%CI) 1.93 (1.50,2.61), NNH 2.3 and remained so post-procedure; $<<0.0001$, RR (95\%) $1.70(1.35,2.23)$, NNH 2.7. Weight at time of study was related to temperature on return to NICU $\left(r^{2}=0.21, p=0.0004\right.$; linear regression). Other demographic factors or cardiorespiratory status did not alter hypothermic risk.

Within the OT group, 93\% of infants were normothermic on departing the NICU (Figure 1A) and $66 \%$ on arrival to the OT (Online Data Supplement Table 1), but only 24\% were normothermic prior to starting surgery; $\mathrm{p}<0.0001$, RR $(95 \% \mathrm{CI}) 3.80(2.33,6.74)$, NNH 1.5 . Compared to the predeparture temperature, infants were a mean $(95 \% \mathrm{CI}) 0.81(0.30,1.33)^{\circ} \mathrm{C}$ (one-way ANOVA) colder prior to starting surgery. At the end of surgery, 59\% were normothermic $(\mathrm{p}=0.0006)$. Externalisation of viscera during surgery did not increase the risk of hypothermia on return to NICU but was associated with increased intra-surgical hypothermia.

Transport to the MRI was not associated with hypothermia (Figure 1B), with $90 \%$ of infants being normothermic on arrival to MRI compared with $86 \%$ prior to departing the NICU (Online Data Supplement Table 2). However, the MRI scan itself was associated with hypothermia, with only 43\% normothermic; $p=0.001$, RR (95\%CI) 2.00 (1.25,3.57), NNH 2.3 (Fisher's exact test). During the MRI scan, core temperature fell by a mean $(95 \% \mathrm{CI}) 0.41(0.16,0.67)^{\circ} \mathrm{C}$ (one-way ANOVA). On return to NICU, $52 \%$ of infants were normothermic; $p=0.04$; RR $(95 \% \mathrm{CI}) 1.64(1.09,2.71), \mathrm{NNH}$ 3.0 . 


\section{DISCUSSION}

In our prospective observational study, we found that infants were at a high risk of developing hypothermia during surgery or MRI, despite being continuously monitored and in a controlled environment. These findings suggest that thermal status needs to be considered during all aspects of an infant's hospital care, not just within the NICU. This study, the first to our knowledge, recorded temperature at different phases of care outside the NICU. Thus, we identified areas in which evidence-based solutions can be targeted, specifically during a MRI scan and in the immediate preoperative period in the OT.

An under-developed thermoregulatory system predisposes sick and pre-term infants to increased hypothermic risk. ${ }^{1,2}$ For this reason, well supported evidence-based thermal management practices exist in the $\mathrm{NICU}^{5,16,17}$ and these translate to high rates of normothermia in the NICU. The finding that transient management in the OT and MRI environments, and transport between the NICU increased the risk of hypothermia to $50 \%$ was not surprising. The innate risks are consistent in both environments, with lower ambient temperatures at $22-25^{\circ} \mathrm{C}$ (similar to the DR). ${ }^{18-20}$ Unlike the NICU and DR, there are few guidelines for thermal care in the OT and MRI. In our study, clinicians were aware of the importance of maintaining normothermia but lacked tested therapeutic options. Transport to and from the OT and MRI was associated with a significant hypothermic risk compared to the NICU environment, except when infants were transported to MRI. Heat loss was exacerbated during either procedure highlighting the need to maintain normothermia in this important clinical phase. The observational study design and sample size limit interpretation of the clinical risks of our findings but similar degrees of hypothermia in the Delivery Room have been shown to increase adverse outcomes. ${ }^{8,9,11,12}$

There is a paucity of literature evaluating neonatal thermal care strategies in the OT. Our study found that the immediate pre-operative period posed the greatest risk for hypothermia. This period is associated with the complex clinical tasks of induction of anaesthesia, airway and vascular instrumentation, often with the infant exposed without an additional heat source. Anaesthetic agents may have also contributed to an increase in the hypothermic risk. Surgical factors, such as type and duration of surgery, infant size and weight, have been suggested as further risks for hypothermia, especially surgery in which a major body cavity such as the abdomen was opened, as seen in our study. ${ }^{21-23}$ Modest gains in normothermia were seen during surgery, although the high variability in temperatures limits clinical interpretation. This may be due to the types of surgery we studied, but more likely reflects the availability of heated humidified gases and the Bair Hugger, a proven intra- 
operative active warming method for patients. ${ }^{14,24}$ The Bair Hugger is difficult to use outside of surgery and is not able to offer the degree of temperature titration needed for infants. The OT feasibly offers the potential to use many active warming solutions validated in the NICU, such as radiant overhead warmers (which may impact on the surgeon's environment) and heating mattresses (which may interfere with diathermy use). ${ }^{25}$ These systems would be practical in the pre-operative period when we observed a significant reduction in the rate of infant normothermia. Our data supports the need for prospective investigation of heated gases and new active warming solutions suited to transport and peri-operative care, including battery-powered and gel warming mattresses.

Conversely, the greatest risk of hypothermia during an MRI was whilst the infant was in the scanner. The immediate pre- and post-procedure period is often shorter and less invasive than surgery. It is not unreasonable to expect infants to become cold during an MRI. Active warming systems are incompatible in the MRI environment which is kept at $22-25^{\circ} \mathrm{C}$ in our institute for technical reasons. As seen in our study, scanning periods are sufficient to allow thermal loss. It is thus surprising that our study is the first to evaluate hypothermic risk in MRI. Previous concerns have been raised regarding the risk of hyperthermia during MRI, due to the occlusive wraps used and heat generated by high output magnets. A recent similar sized study of 25 infants reported no hyperthermic events, and four instances of hypothermia during a 3T MRI scan $(<60 \mathrm{~min}){ }^{26}$ The authors also noted that temperature upon entering the scan related to exit temperature. Together, these findings suggest temperature monitoring with the option of active warming to ensure normothermia at the start of an MRI scan could benefit neonatal patients. Whether the use of passive thermal systems, such as an occlusive polyurethane bag, ${ }^{9,11,18,27}$ would improve outcomes by preventing heat loss during the MRI warrants investigation.

Body temperature on arrival to the NICU impacts post-procedural NICU care, with hypothermia predominating $15 \mathrm{~min}$ after reinstated NICU thermal care. It is likely this time point was too short to allow active warming methods to be effective. Although the sample size may have been too small to make meaningful conclusions, the observation that normothermia rates were higher by $60 \mathrm{~min}$ suggests effective care. The differences in normothermia rates between the OT and MRI groups 60 min after returning to the NICU were unexpected. This may be related to the need for higher dependency NICU care after surgery. Future studies should consider later post-procedure end-points to better elucidate rewarming trajectories in different levels of care.

Our pragmatic observational study design limits interpretation. However, we contend that our study design was necessary due to the knowledge gap limiting causative and clinical hypotheses to be 
generated. The awareness that a temperature was being recorded potentially introduced bias, although this is likely to result in normothermia. The ability to accurately measure infant core temperature during MRI would have been useful. Although an experienced clinician always performed probe placement, incorrect placement may influence temperature data. The limited use of oesophageal core temperature also precludes robust analysis of the effect of monitoring site. Our study generally involved term or near-term infants. Preterm infants, a population extensively studied in the DR, are likely to have different thermal risks. In particular, the transport periods may be sufficient to cause hypothermia. In our unit, preterm infants are more likely to have surgery on the NICU itself and to have a planned MRI deferred to term corrected age compared to infants born at full term. Finally, caution should be used in generalising our data to centres with different physical factors, such as ambient room temperatures and transport distance and time, emphasising the need for clinicians to review their own centre practices and risks.

\section{Conclusions}

Infants experience significant heat loss during management outside of the NICU, specifically in the peri-operative period and during an MRI scan. Our study provides confirmation that evidence-based guidelines to manage temperature using active and passive systems are needed.

Acknowledgements: A/Prof Susan Donath of the Centre of Epidemiology and Biostatistics Unit (Murdoch Children's Research Institute) for her advice on the study design and statistical analysis. 


\section{REFERENCES}

1. Knobel RB. Fetal and Neonatal Thermal Physiology. Newborn Infant Nurs Rev 2014;14:45-9.

2. Knobel-Dail RB. Role of effective thermoregulation in premature neonates. Res Rep Neonatol 2014:147.

3. Çınar ND, Filiz TM. Neonatal thermoregulation. J Neonatal Nurs 2006;12:69-74.

4. Tansey EA, Johnson CD. Recent advances in thermoregulation. Adv Physiol Educ 2015;39:139-48.

5. Knobel RB. Thermal Stability of the Premature Infant in Neonatal Intensive Care. Newborn Infant Nurs Rev 2014;14:72-6.

6. Ellis J. Neonatal hypothermia. J Neonatal Nurs 2005;11:76-82.

7. Bastug O, Gunes T, Korkmaz L, et al. An evaluation of intra-hospital transport outcomes from tertiary neonatal intensive care unit. J Matern Fetal Neonatal Med 2016;29:1993-8.

8. DeMauro SB, Douglas $\mathrm{E}$, Karp $\mathrm{K}$, et al. Improving delivery room management for very preterm infants. Pediatrics 2013;132:e1018-25.

9. Vohra S, Roberts RS, Zhang B, et al. Heat Loss Prevention (HeLP) in the delivery room: A randomized controlled trial of polyethylene occlusive skin wrapping in very preterm infants. $J$ Pediatr 2004;145:750-3.

10. Vohra S, Reilly M, Rac VE, et al. Study protocol for multicentre randomized controlled trial of HeLP (Heat Loss Prevention) in the delivery room. Contemp Clin Trials 2013;36:54-60.

11. Smith J, Usher K, Alcock G, et al. Application of plastic wrap to improve temperatures in infants born less than 30 weeks gestation: a randomized controlled trial. Neonatal Netw 2013;32:235-45.

12. Kent AL, Williams J. Increasing ambient operating theatre temperature and wrapping in polyethylene improves admission temperature in premature infants. J Paediatr Child Health 2008;44:325-31.

13. Paley MN, Hart AR, Lait M, et al. An MR-compatible neonatal incubator. $\mathrm{Br} J$ Radiol 2012;85:952-8.

14. Hart SR, Bordes B, Hart $\mathrm{J}$, et al. Unintended perioperative hypothermia. Ochsner $J$ 2011;11:259-70.

15. Morehouse D, Williams L, Lloyd C, et al. Perioperative hypothermia in NICU infants: its occurrence and impact on infant outcomes. Adv Neonatal Care 2014;14:154-64.

16. Turnbull V, Petty J. Evidence-based thermal care of low birthweight neonates. Part one. Nurs Child Young People 2013;25:18-22. 
17. New K, Bogossian F, East C, et al. Practice variation in the transfer of premature infants from incubators to open cots in Australian and New Zealand neonatal nurseries: results of an electronic survey. Int J Nurs Stud 2010;47:678-87.

18. McCall EM, Alderdice F, Halliday HL, et al. Interventions to prevent hypothermia at birth in preterm and/or low birthweight infants. Cochrane Database Syst Rev 2010(3):CD004210 doi: 10.1002/14651858.CD004210.pub4.

19. Singh A, Duckett J, Newton T, et al. Improving neonatal unit admission temperatures in preterm babies: exothermic mattresses, polythene bags or a traditional approach? J Perinatol 2010;30:45-9.

20. Laptook AR, Watkinson M. Temperature management in the delivery room. Semin Fetal Neonatal Med 2008;13:383-91.

21. Qureshi M. Prevention of hypothermia in surgical neonates in operation theatre: recommendations of neonatal surgery research group. J Neonatal Surg 2012;1:12.

22. Tander B, Baris S, Karakaya D, et al. Risk factors influencing inadvertent hypothermia in infants and neonates during anesthesia. Paediatr Anaesth 2005;15:574-9.

23. Thomas J. Reducing the risk in neonatal anesthesia. Paediatr Anaesth 2014;24:106-13.

24. John M, Ford J, Harper M. Peri-operative warming devices: performance and clinical application. Anaesthesia 2014;69:623-38.

25. McCarthy LK, Molloy EJ, Twomey AR, et al. A randomized trial of exothermic mattresses for preterm newborns in polyethylene bags. Pediatrics 2013;132:e135-41.

26. Cawley P, Few K, Greenwood R, et al. Does Magnetic Resonance Brain Scanning at 3.0 Tesla Pose a Hyperthermic Challenge to Term Neonates? J Pediatr 2016;175:228-30.

27. Ibrahim CP, Yoxall CW. Use of plastic bags to prevent hypothermia at birth in preterm infants-do they work at lower gestations? Acta Paediatr 2009;98:256-60.

This article is protected by copyright. All rights reserved. 


\section{FIGURE LEGENDS}

Figure 1: Core temperature $\left({ }^{\circ} \mathrm{C}\right)$ at each study phase for the OT (A) and MRI (B) groups. Grey shaded region indicates normothermic range $\left(36.5^{\circ} \mathrm{C}-37.5^{\circ} \mathrm{C}\right)$. All data mean and SD. Rates of normothermia at each phase (\%) shown at top of each error bar. NICU $\mathrm{D}_{\mathrm{D}}$; Depart NICU, OT; Arrive at OT, Surg ; Start Surgery, Surg ${ }_{E}$; End Surgery, MRI ; Enter MRI, MRI $I_{L}$; Leave MRI, NICUR; NICU Return, NICU ${ }_{R+15}$; NICU Return $(+15 \mathrm{~min}) .{ }^{*} \mathrm{p}<0.05, \# \mathrm{p}=0.01, \dagger \mathrm{p}=0.04, \ddagger \mathrm{p}=0.01$ compared to $\mathrm{NICU}_{\mathrm{D}}$ (one-way ANOVA with Tukey post-tests).

This article is protected by copyright. All rights reserved. 
Table 1: Infant characteristics at study enrolment

\begin{tabular}{|c|c|c|c|}
\hline Mean (SD), median (range) or $\mathbf{n}(\%)$ & All infants $(n=62)$ & OT Group $(n=41)$ & MRI Group (n=21) \\
\hline Gestational Age at birth (completed weeks) & $36(4)$ & $35(4)$ & $38(2)^{*}$ \\
\hline $\begin{array}{l}\text { Corrected Gestational Age at procedure } \\
\text { (completed weeks) }\end{array}$ & $39(4)$ & $39(4)$ & $39(3)$ \\
\hline Age at study (days) & $15(0,134)$ & $25(0,134)$ & $7(1,45)^{*}$ \\
\hline Birthweight (g) & $2591(844)$ & $2393(937)$ & $3063(559)^{*}$ \\
\hline Weight at study (g) & $3010(702)$ & $2961(777)$ & $3160(633)$ \\
\hline Gender ( $\%$ females) & $26(42 \%)$ & $16(39 \%)$ & $10(48 \%) \dagger$ \\
\hline Time away from NICU (min) & & $185(34,355)$ & $69(45,106)^{*}$ \\
\hline $\begin{array}{l}\text { Respiratory Support via an endotracheal } \\
\text { tube }^{\dagger}\end{array}$ & $41(66 \%)$ & $41(100 \%)$ & $0(0 \%)$ \\
\hline Primary organ system involved (all infants) & \multicolumn{3}{|c|}{$\begin{array}{l}\text { GIT }(n=21) \text {, CNS }(n=17) \text {, Renal }(n=2) \text {, ENT }(n=4) \text {, OA }(n=2) \text {, Chest/Thorax } \\
(n=2) \text {, Congenital }(n=5) \text {, Skeletal }(n=1) \text {, Respiratory }(n=3) \text {, Unknown }(n=1) \text {, } \\
\text { Metabolic }(n=4)\end{array}$} \\
\hline Type of surgery (OT Group) & \multicolumn{3}{|c|}{$\begin{array}{l}\text { GIT }(n=24) \text {, CNS }(n=2) \text {, Renal }(n=2) \text {, ENT }(n=7) \text {, OA }(n=1) \text {, Chest/Thorax } \\
(n=5)\end{array}$} \\
\hline
\end{tabular}

Abbreviations: GIT; Gastrointestinal Tract, CNS; Central Nervous System, ENT; Ear, Nose and Throat, OA; Oesophageal Atresia ${ }^{*} p<0.05$ (compared to OT Group) unpaired Welch's corrected t-test or Mann-Whitney test, and $\dagger$ Fisher's exact test.

${ }^{\dagger}$ Respiratory status was unchanged during the study period.

This article is protected by copyright. All rights reserved. 
Table 2: Normothermia and hypothermia during each study phase

\begin{tabular}{|c|c|c|c|c|c|}
\hline \multirow{2}{*}{$\mathbf{2}$} & \multicolumn{5}{|c|}{ Phase } \\
\cline { 2 - 6 } & Depart & Pre- & Post- & NICU & NICU return \\
& NICU & procedure & procedure & return & +15 min \\
\hline Normothermia & $90 \%$ & $47 \%$ & $53 \%$ & $52 \%$ & $45 \%$ \\
\hline Hypothermia & $10 \%$ & $53 \%$ & $47 \%$ & $48 \%$ & $55 \%$ \\
\hline RR (95\%) & & & & & \\
\hline
\end{tabular}

RR; Relative Risk (Fisher's exact test) 


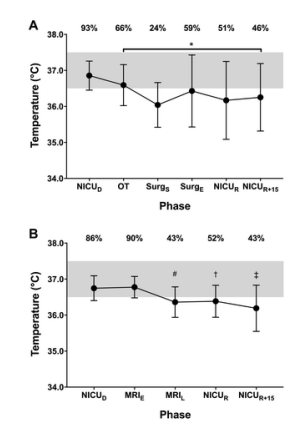

TENTS Figure 1.tiff

This article is protected by copyright. All rights reserved. 


\section{University Library}

\section{- M M N E R VA A gateway to Melbourne's research publications}

Minerva Access is the Institutional Repository of The University of Melbourne

\section{Author/s:}

Paul, JMD;Perkins, EJ;Pereira-Fantini, PM;Suka, A;Farrell, O;Gunn, JK;Rajapaksa, AE;Tingay, DG

Title:

Surgery and magnetic resonance imaging increase the risk of hypothermia in infants

Date:

2018-04-01

\section{Citation:}

Paul, J. M. D., Perkins, E. J., Pereira-Fantini, P. M., Suka, A., Farrell, O., Gunn, J. K., Rajapaksa, A. E. \& Tingay, D. G. (2018). Surgery and magnetic resonance imaging increase the risk of hypothermia in infants. JOURNAL OF PAEDIATRICS AND CHILD HEALTH, 54 (4), pp.426-431. https://doi.org/10.1111/jpc.13824.

Persistent Link:

http://hdl.handle.net/11343/283472 